\title{
Microsurgical Resection of Multiple Giant Glomus Tumors
}

\author{
Guilherme H. W. Ceccato ${ }^{1}$ Marcio S. Rassi ${ }^{2}$ Luis A. B. Borba ${ }^{3,4}$ \\ 1 School of Medicine, Federal University of Paraná, Curitiba, PR, Brazil \\ 2 Department of Neurosurgery, A.C. Camargo Cancer Center, \\ São Paulo, Brazil \\ ${ }^{3}$ Department of Neurosurgery, Evangelic University Hospital of \\ Curitiba, PR, Brazil \\ ${ }^{4}$ Department of Neurosurgery, Federal University of Paraná, Curitiba, \\ PR, Brazil
}

\author{
Address for correspondence Luis A. B. Borba, MD, PhD, International \\ Fellow of the American Association of Neurological Surgeons, Rua \\ Paulo Gorski 1240 \#7, CEP 81210220, Curitiba, PR, Brazil \\ (e-mail: luisborba@me.com).
}

J Neurol Surg B 2019;80(suppl S4):S385-S388.

Glomus tumors, also called paragangliomas, are challenging lesions, demanding accurate knowledge of complex anatomy and pertinent approaches. We present the case of a 39year-old male presenting with headache, vertigo, tinnitus, hearing loss, and hoarseness. Neurological assessment showed facial paralysis House-Brackmann IV and lower cranial nerves deficits. Preoperative magnetic resonance imaging (MRI) demonstrated two large lesions, suggestive of a glomus jugulare, and carotid body paragangliomas. Considering worsening of the symptoms and the important mass effect of both lesions over the neurovascular structures, microsurgical excision was offered, after preoperative tumor embolization. We preferred to approach both lesions in the same operation, starting by the cervical tumor. Initially there was not an easily identifiable dissection plane between the tumor and the carotid artery, but it was achieved after performing a subadventitial dissection, being possible to resect the entire lesion. The jugular foramen lesion was approached through a postauricular transtemporal approach, skeletonizing the sigmoid sinus, jugular bulb, and facial nerve, following a complete mastoidectomy. The tumor, extending to the intradural compartment, middle ear, internal auditory canal, petrous internal carotid artery, and internal jugular vein was completely removed. Postoperative MRI demonstrated complete resection of both lesions, and pathology confirmed to be paragangliomas. In the immediate postoperative period, the facial paralysis evolved to House-Brackmann grade VI, improving to grade III during follow-up. The patient underwent a vocal cord medialization in order to improve voice quality and swallowing. These are challenging lesions and extensive laboratory training is mandatory to be familiarized with the regional anatomy and its various surgical approaches.

The link to the video can be found at: https://youtu.be/gA_ckwFq_9c.

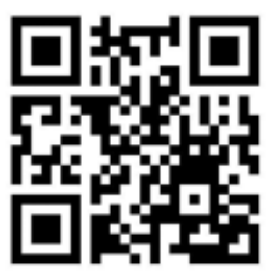

received

April 1, 2019

accepted after revision

July 7, 2019

published online

October 3, 2019

www.thieme.com/skullbasevideos

www.thieme.com/jnlsbvideos

DOI https://doi.org/

10.1055/s-0039-1695055.

ISSN 2193-6331.
๑) 2019 Georg Thieme Verlag KG
Stuttgart · New York

License terms

(c) (1) $\ominus$ (\$) 


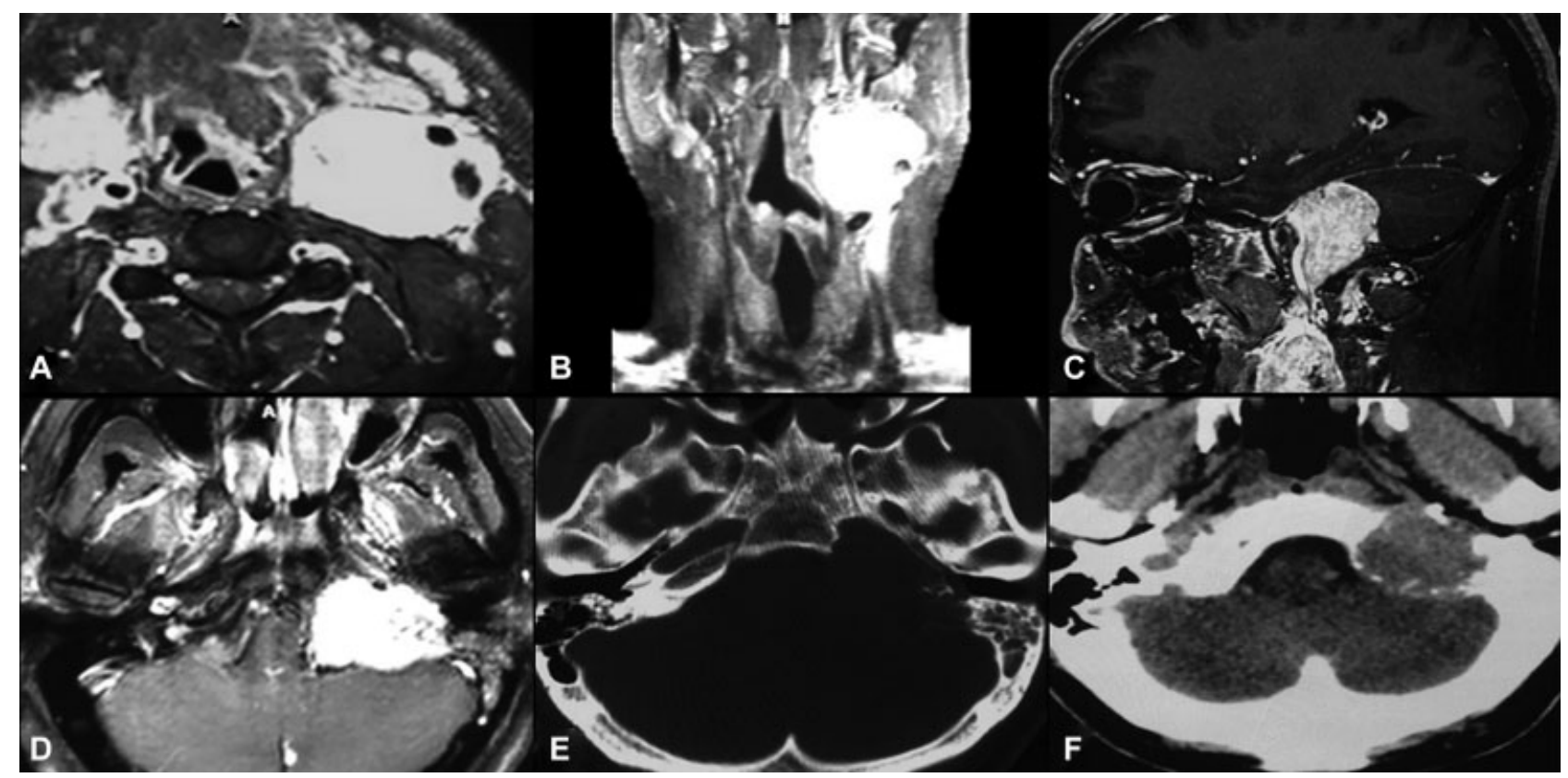

Fig. 1 Preoperative imaging. (A) axial and (B) coronal MRI slices demonstrating the lesion involving the left carotid bifurcation and the internal and external carotid arteries. (C) Sagittal post contrast MRI demonstrating the cervical lesion associated with the carotid bifurcation and other related to the jugular foramen. (D) Axial MRI demonstrating intradural extension toward the cerebellopontine angle. (E) Bone window CT scan with compromise of the left carotid canal, middle ear and internal auditory canal besides the jugular foramen. (F) Brain window CT scan demonstrating a mass in the left jugular foramen. $\mathrm{CT}$, computed tomography; MRI, magnetic resonance imaging.

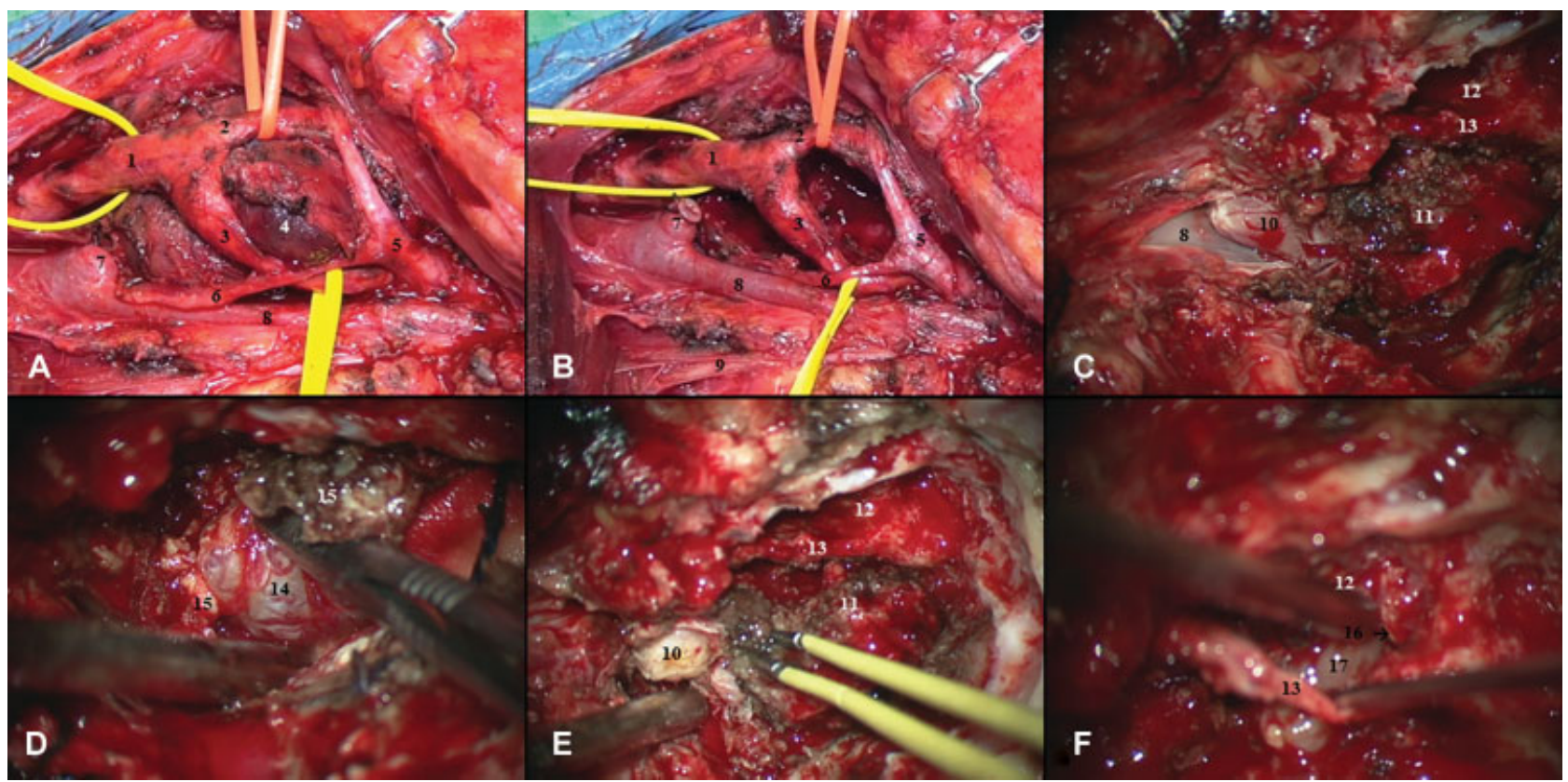

Fig. 2 Intraoperative images. (A) Exposure of the carotid body paraganglioma in the carotid bifurcation. (B) Surgical field after resection of the carotid body paraganglioma. (C) Extradural part of the glomus jugulare tumor extending inside the internal jugular vein. (D) Detachment of the tumor from inside the jugular vein, remaining connected to the extradural part of the paraganglioma. (E) Removal of the intradural extension of the lesion, including resection of the compromised dura mater. (F) Resection of the tumor extending from the tympanic cavity toward the internal auditory canal. 1, common carotid artery; 2, external carotid artery; 3, internal carotid artery; 4, carotid body paraganglioma; 5 , hypoglossal nerve (CN XII); 6, branch to the ansa cervicalis from the hypoglossal nerve; 7, facial vein; 8- Internal jugular vein; 9 , accessory nerve (CN XI); 10, glomus jugulare paraganglioma extending inside the internal jugular vein; 11, extradural aspect of the glomus jugulare paraganglioma; 12, tympanic cavity; 13, facial nerve (CN VII); 14, intradural extension of the tumor; 15, compromised dura mater; 16 , internal auditory canal; 17 , tumor extension toward the internal auditory canal. 
Conflict of Interest

None declared.

\section{Disclosure statement}

All authors contributed to this article and attest to no conflicts of interest. All authors have no financial disclosures.
Patient Consent Statement

Informed consent was obtained from the patient for publication of this operative video. 